\title{
Evaluation of Setup Uncertainty in Radiotherapy of Pelvic Cancer
}

\author{
Mohamed Sayed ${ }^{* 1}$ MSc.;AhmedAl-Agamawi ${ }^{1}$ MD; Khaledelshahat ${ }^{1} \mathrm{PhD}$; \\ WaelEl-Sheshtawy ${ }^{1} \mathrm{MD}$.
}

*Corresponding Author:

Mohamed Sayed

mohamedsayed15984@gmail.com

Received for publication May5, 2020; Accepted July2, 2020; Published onlineAugust 26, 2020.

Copyright 2020 The Authors published by Al-Azhar University, Faculty of Medicine, Cairo, Egypt. All rights reserved. This an open-access article distributed under the legal terms, where it is permissible to download and share the work provided it is properly cited. The work cannot be changed in anyway or used commercially.

doi:10.21608/aimj.2020.28802.1209

${ }^{1}$ Clinical Oncology and Nuclear Medicine Department, Faculty of Medicine, Al-Azhar University, Cairo, Egypt.

\begin{abstract}
Background:The most important event of RT (Radiation Therapy)is to dependably expand the positions to the target volume while limiting the side effects to the normal tissues. Thusly, day by day treatment arrangement is considered as a basic necessity in RT (Radiation Therapy) for an exact positions and setup accuracy for treatmentbut still the Set-up errors are the inevitable features of the radiation therapy RT (Radiation Therapy) process.

Aim of work:To evaluate three dimensional set-up errors and propose optimum margins for target volume coverage inthe pelvic radiation therapy.

Patient and Methods: This study aiming to evaluate setup uncertainty related to pelvic cancer radiotherapy in the Clinical Oncology and Nuclear Medicine Department, at Al Hussein hospital, Al-Azhar University and to estimate how large planning target volume margins are needed in the Pelvic cancer cases.

Results: the mean variation for action level for plan is $3.3 \mathrm{~mm}$ (1) SD (Standard Deviation) for pelvic cancer cases. The greatness of precise and irregular mistakes for pelvic cancer cases in our investigation are comparable or not exactly different examinations. To contrast the outcomes and other distributed investigation, we have considered PTV $(90,95$,$) margin. In our investigation, the determined CTV-PTVClinical$ Target Volume- Planning Target Volume) edge for pelvic cancer patient in the vertical, longitudinal and Lateral Variations from iso-center position were $4.12 \mathrm{~mm}, 4.62 \mathrm{~mm}$ and $3.23 \mathrm{~mm}$, individually.

Conclusion: The consequences of this examination are distinctive to the discoveries of current work. In the present work, we have changed the isocenter in the fourth portion, which brought about decrease of the efficient mistakes. Another distinction can be ascribed to the recurrence of online confirmation.

Keywords:Setupuncertainty; Pelvic cancer; Radiotherapy.
\end{abstract}

Disclosure: The authors have no financial interest to declare in relation to the content of this article. The Article Processing Charge was paid for by the authors.

Authorship: All authors have a substantial contribution to the article.

\section{INTRODUCTION}

The point of Radiation Treatment (RT) is to dependably expand the portion to the objective while limiting the poisonous quality to the typical tissues. Thusly, day by day treatment arrangement is considered as a basic necessity in RT for an exact portion conveyance. The arranging planning target volume (PTV) is characterized as the clinical target volume (CTV) in addition to an edge to represent understanding situating vulnerabilities, bar arrangement and organ movement (for example arrangement edge and interior edge). Arrangement edges directly affect the inclusion of target volume. Hence, these ought to be upgraded to forestall incidental light of Organ AtRisks (OARs). ${ }^{1,2}$

Arrangement vulnerabilities can be isolated into two classes: efficient blunders and arbitrary errors.
Whereas the irregular mistakes obscure the portion dispersion, the methodical part of blunders prompts amove of the aggregate portion appropriation comparative with the objective. The efficient blunders are reproducible predictable mistakes, happening in a similar bearing and extent yet arbitrary (everyday) blunders can change in course, size and are eccentric. The methodical blunders as opposed to the irregular mistakes are increasingly perilous in light of the fact that they influence all treatment meetings. In this way, methodical mistakes may prompt the repeat of the tumor or genuine harm in ordinary organs.

The board accessibility of EPIDs (Electronic Portal Image Devices) has prompted it be a powerful apparatus to diminish arrangement mistakes. PretreatmentEPIs(Electronic Portal Images) give the assessment of arrangement blunders. ${ }^{4,5}$ Right now, 
blunders assessment is doing utilizing an entrance picture and a carefully recreated radiograph DRR (DigittalyReconstructed Radiographes). The goal of this examination was to measure the difference between fragmentary arrangement blunders and 3D vector lengths and compute CTV-PTV edge for various treatment destinations, for pelvic cancercases includingprostate cancercases by electronic entrance pictures direction and decide the ideal PTV edges.

\section{PATIENTS AND METHODS}

\section{Patients Selection:}

This study aiming to evaluate Setup Uncertainty related to Pelvic Cancer Radiotherapy in the Clinical Oncology and Nuclear Medicine Department, at Al Hussein Hospital of Al-Azhar University and to estimate how large planning target volume margins are needed in the Pelvic Cancer Radiation Therapy when the 2D imaging is used for frequent setup verification in the period betweenFeb. 2016 to Feb.2020 including 101 patients with different types of pelvic cancer that treated with Raiotherapy.

Treatment Simulation and Planning:

At our establishment, pelvic cancer patients didn't utilize immobilization only but also utilizing the embedded 3 fiducially lead markers. The pelvic cancer patients needed to exhaust rectum and had a full bladder reenactment and treatment meetings before figured tomography CT (Computed Tomography) arranging and day by day treatment. For all pelvic cancer patients were examined in head first recumbent position with laser pillars direction in CT plan for pelvic cases, EPIs were performed for the initial 3 successive treatment divisions. Online arrangement mistake amendment was accomplished for these three portions. At the fourth division, patients were moved to the new iso-center, with normal removals in the initial three parts and were followed once week by week from that point.

The whole study group going to receive pelvic radiation therapyThere are multiple factors which can lead to setup uncertainties for the pelvic and prostate cases. Target volume position in pelvic and prostate cancer can change owing to intestinal movement and vary filling in the bladder and rectum ${ }^{5-7}$.

Localization, Immobilization, and Simulation: During simulation and treatment, the patient is prone with a full bladder to decrease the small intestinal dose, rectal markers are used during simulation. The laser localizer system is used to put a 3 radio opaque marks for position reproducibility, these marks are used as references for repositioning of the patient for treatment delivery. The patient then scanned by CT scan machine from the ischialtuberosities up to the lower border of L5 with cuts interval $5 \mathrm{~mm}$.

In order to make a dosimetric comparison, IMRT treatment plans were generated for selected patients. Acute effects and late effects in addition to potential complications of treatment were explained clearly toall patient and the study was approved by the university ethics committee. Varian - eclipse planning system was used as an algorithm for planning. Body CTcontour was generated automatically and tareget volumes with the surrounding critical structures were contoured manually. CTV (Clinical Target Volume) was created on the plan then PTV (Planning Target Volume) was created by a $10 \mathrm{~mm}$ expansion around CTV in all directions. Target and critical organ delineations were performed by the same radiation oncologist. To perform Dosimetric evaluation for treatment plan in terms of: Planning target volume (PTV) coverage, Organs at risk (OAR) dose, Conformity index (CI)\&Homogeneity index (HI).

At that point, the online arrangement mistake amendment would apply if remedy was required. Prior to treatment, patients were positioned with a suitable immobilization device when possible. Then, they were setup to treatment room by laser and skin marks. Portal images are obtained for the first two fractions for the pelvic cancer patients with Hip Fix thermoplastics when needed. Displacements between Digitally Reconstructed Radiographs (DRR) and Electronic Portal Images (EPI) are estimated and analyzed along major three axes. Mean displacements, population systematic and random errors and three dimensional vectors of displacements are calculated.

For example patients with prostate cancer, EPIs were completed a few times each week since we needed to assess the adequacy of fiducial marker-based position check during pelvis EBRT just because at our oncology departement. Hence, online arrangement blunder revision was done for pelvic cancer patients. Coordinating DRRs and entrance pictures were performed utilizing the life structures coordinating programming system.

For study the arrangement blunders, the dislodging in two translational ways were surveyed in each field. The symmetrical entry pictures were coordinated utilizing the unmistakable hard tourist spots with their separate DRRs. The activity level was the relocation more noteworthy than $3 \mathrm{~mm}$ in pelvic cancer cases along one course where Cut thickness was $5 \mathrm{~mm}$ in all cases for CT planning with considering the treatment Iso-Center. At that point, new entryway pictures were obtained. Persistent arrangement blunders were evaluated along three translational bearings $\{$ (vertical $(\mathrm{Y})$, longitudinal $(\mathrm{Z})$ and horizontal (X\}).

\section{Measurements:}

The deliberate and arbitrary mistakes were determined utilizing the dislodging in three translational ways. For pelvic cancer cases, the orderly blunders were characterized as deviations between the arranged patient position and original patient situation of initial three back to back treatment divisions. The arbitrary blunders were characterized as deviations between various treatment portions taken week after week during a course of the treatment. Standard deviation (SD) of the orderly blunders $(\Sigma)$ and SD of the irregular mistakes $(\sigma)$ were dissected. Forexamble, prostate cancer patients, $\Sigma$ alludes to SD of every individual 
mean, and $\sigma$ is resolved through the root mean square of the individual SD of all patients. ${ }^{3}$ Likewise, we measured the recurrence of $3 \mathrm{D}$ vector lengths and determined the greatness of 3D vector utilizing.

Follow UP during treatment sessions:

Portal images were acquired at a dose rate 400 monitor unit (MU) per minute and 1 MU were delivered per field for portal acquisition. EPID images were compared to the DRRs (as a reference image) created for the orthogonal portals at $0^{\circ}$ (anterior) and $90^{\circ}$ (lateral) using treatment planning software (TPS).

Observing of patient situating can be performed by EPID. Matching DRRs and portal images were performed using the anatomy matching software (ARIA-record \& verify system).For study the setup errors, the displacement in two translational directions were assessed in each field. The orthogonal portal images were matched using the visible bony landmarks with their respective DRRs.

Accordingly, any adjustments in the treatment isocenter will be rectified. In the present investigation, we assessed the between partial set up mistakes for different treatment locales of 101 patients utilizing EPID. For the pelviccnacer cases, about $84 \%, 77 \%$ and $77 \%$ of the arrangement relocations were under $3.52 \mathrm{~mm}$ in the vertical, longitudinal and horizontal headings, individually.

There are different elements which can prompt arrangement vulnerabilities for the pelvic cancer cases. Target volume position in pelvic malignancy can change attributable to intestinal development and differ filling in the bladder and rectum. ${ }^{8.9}$ In the meantime, skin imprints can undoubtedly move in these treatment locales, and can prompt arrangement blunder. ${ }^{8.9}$ Utilizing flimsy lines on the patient's skin just as great modified skin fiducial markers and exactness of laser room can decrease arrangement deviations in theories treatment destinations.

\section{RESULTS}

The overall mean (average) errors are calculated for all the patients over all whole period of treatment as shown in table1.

Table 1: The Calculated Mean Error (mm) in the Anterior Field \& the Lateral Field for 101 Patients during all sessions (444portal images)

\begin{tabular}{|l|l|l|l|}
\hline Direction & VRT & LONG & LAT \\
\hline $\begin{array}{l}\text { Mean Error } \\
\text { (cm) }\end{array}$ & -0.00045 & -0.00583 & -0.17578 \\
$\begin{array}{l}\text { Anterior } \\
\text { Field\} }\end{array}$ & & & \\
\hline $\begin{array}{l}\text { Mean Error } \\
\text { (cm) }\end{array}$ & -0.32735 & 0.041256 & -0.0009 \\
\hline \begin{tabular}{l} 
Lateral Field\} \\
\hline
\end{tabular} & & & \\
\hline
\end{tabular}

The Calculated Systematic Error (mm) and Random Error (mm) for 101 Patient during all sessions as shown in table2over all whole period of treatment.

\begin{tabular}{|c|c|c|c|}
\hline Direction & VRT & LONG & LAT \\
\hline $\begin{array}{c}\text { Systematic Error } \\
(\mathrm{mm})\end{array}$ & 0.94 & 1.23 & 1.52 \\
\hline $\begin{array}{c}\text { Random Error } \\
(\mathrm{mm})\end{array}$ & 1.56 & 2.05 & 1.89 \\
\hline
\end{tabular}

Table 2: The Calculated Systematic Error (mm) and Random Error (mm) for 101 Patient during all sessions

The variation in different directions for each patient for iso-center position are shown in figure 1.

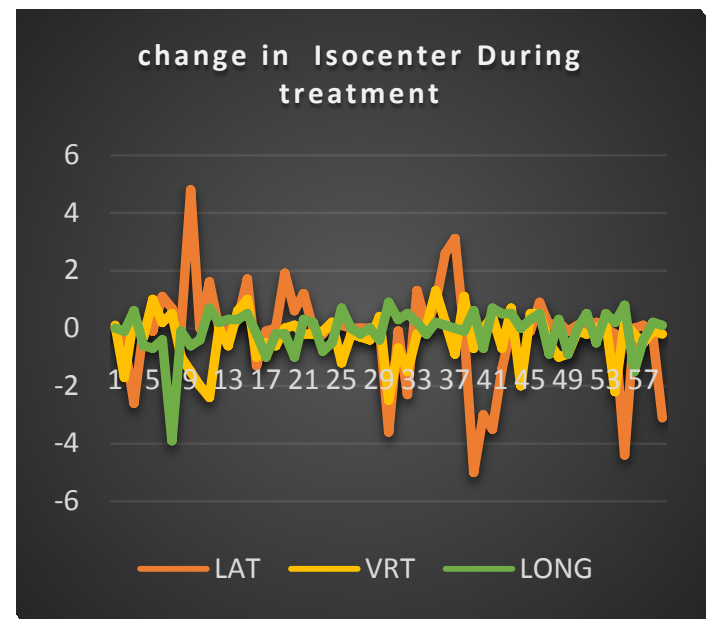

Fig. 1: the variation in different directions for each patient for iso-center position.

The Mean deviation of pelvis shift in different directions during all treatment sessions for 101 patients by 222 portal images for Vertical $(\mathrm{cm})$, Longitudinal $(\mathrm{cm})$ and Lateral $(\mathrm{cm})$ directions in the Anterior field as presented respectively in Figures 2 ,3\&4.

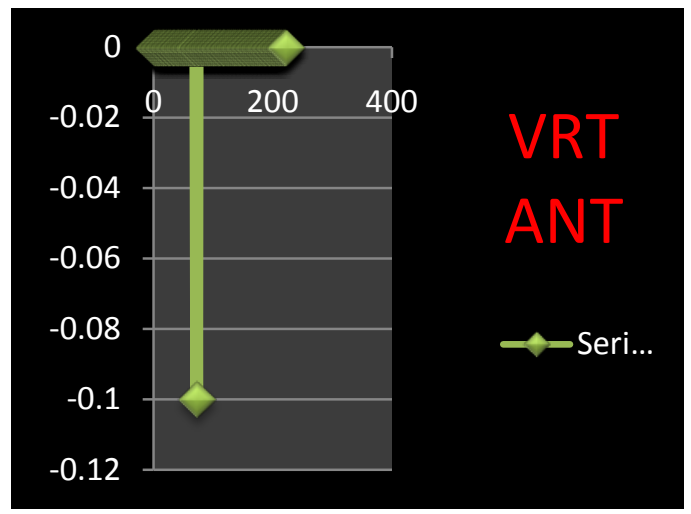

Figure 2:Scale by $\mathrm{cm}$ for variations in Vertical direction in the Anterior field 


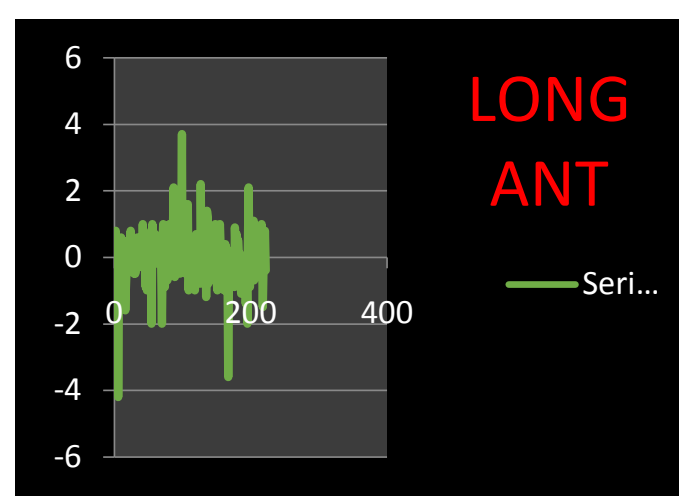

Figure 3:Scale by $\mathrm{cm}$ for variations in Longitudinal direction in the Anterior field

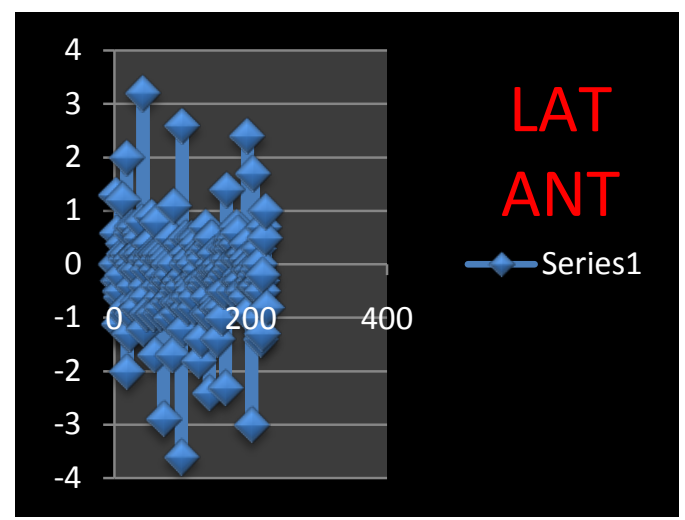

Figure 4:Scale by $\mathrm{cm}$ for variations in Lateral direction in the Anterior field

The Mean deviation of pelvis shift in different directions during all treatment sessions for 101 patients by 222 portal images for Vertical (cm), Longitudinal $(\mathrm{cm})$ and Lateral $(\mathrm{cm})$ directions in the Lateral field as presented respectively in Figure 5, $6 \& 7$.

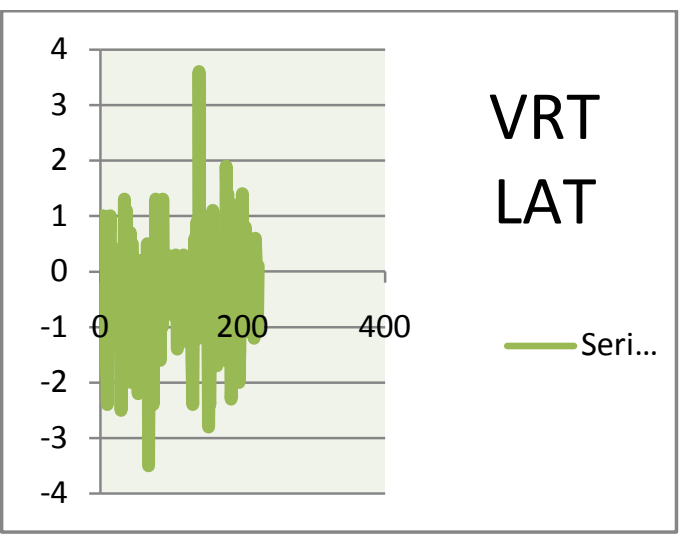

Figure 5:Scale by $\mathrm{cm}$ for variations in Vertical direction in the Lateral field

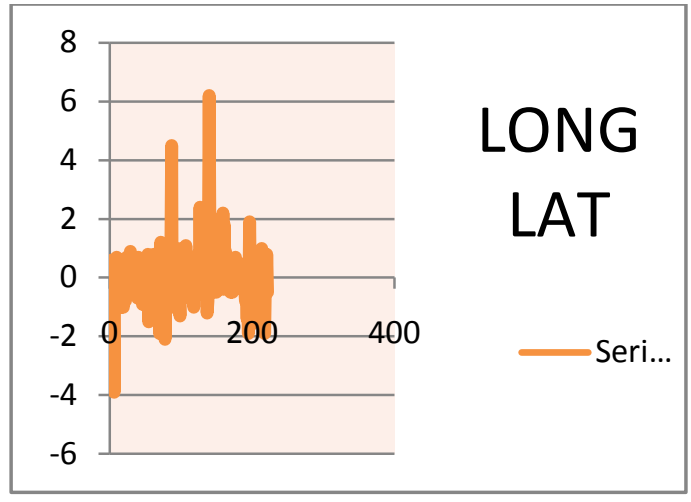

Figure 6: Scale by $\mathrm{cm}$ for variations in Longitudinal direction in the Lateral field

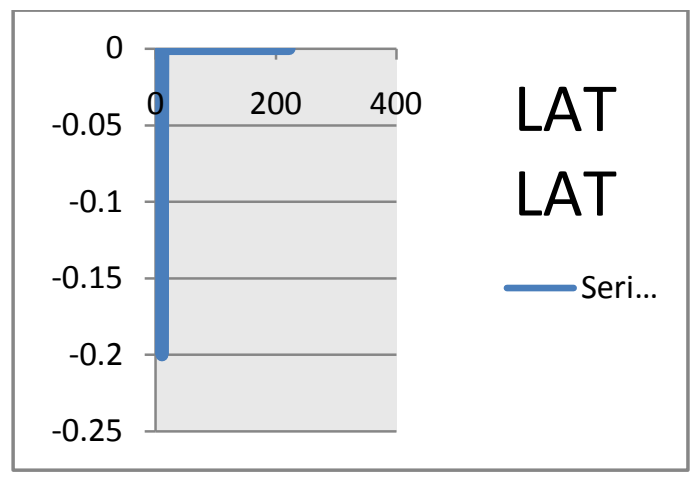

Figure 7:Scale by $\mathrm{cm}$ for variations in Lateral direction in the Lateral field

The Confidence level for PTV margins (mm)are summarized in table 3.

\begin{tabular}{|c|r|r|r|r|r|r|r|r|r|}
\hline $\begin{array}{c}\text { Confidence } \\
\text { level }\end{array}$ & \multicolumn{3}{|c|}{ VRT } & \multicolumn{3}{c|}{ LAT } & \multicolumn{3}{c|}{ LONG } \\
\hline Dose & 90 & 95 & 99 & 90 & 95 & 99 & 90 & 95 & 99 \\
\hline 99 & 3.1 & 3.0 & 4.52 & 4.1 & 4.82 & 4.8 & 4.2 & 4.5 & 4.7 \\
\hline 95 & 3.5 & 3.4 & 4.10 & 3.5 & 4.25 & 5.32 & 3.8 & 4.12 & 4.85 \\
\hline 90 & 4.1 & 3.62 & 3.85 & 4.6 & 4.95 & 5.10 & 3.2 & 3.85 & 5.13 \\
\hline 85 & 4.3 & 3.22 & 3.6 & 4.56 & 5.12 & 4.92 & 4.6 & 3.85 & 5.18 \\
\hline
\end{tabular}

Table 3 : Confidence level for PTV margins (mm)

\section{DISCUSSION}

The distributions of the translational and rotational variations in each direction as well as the total displacement of the treatment iso-center from the simulation iso-center were determined. From above results and analysis of 444 portal images of the all 101 patients revealed average unidirectional translational deviations of less than $2.6 \mathrm{~mm}$ and a standard deviation of $5.4 \mathrm{~mm}$. The average total undirected distance between the treatment and simulated iso-centers was $6.7 \mathrm{~mm}$ with a standard deviation of $5 \mathrm{~mm}$.

Individual for each patient analysis revealed 16 patients from all patients had statistically significant nonzero mean translational variations ( $\mathrm{p}<0.05$ ). 
Translational variations measured with film were an average of $1.8 \mathrm{~mm}$ less than those measured with EPID, but this difference was not statistically significant. This may explain the slightly larger average translational variations observed with EPID vs. film, and suggests that the use of EPIDs is a superior method for assessing the true extent of setup displacements. Although no statistically significant translational variations for the patient group overall were observed, $90 \%$ of patients had significant translational variations in at least one direction when analyzed separately. (film is used as verification system for iso-center variations in old patient before using portal imager as setup verification system)

We found that in the all treatment locales, the arbitrary mistakes were more noteworthy the sidelong way contrasted with two different bearings. This is most likely because of the optical figment and the mistake in coordinating laser and line on the patient body.

The Action is $3.3 \mathrm{~mm}$ (1SD) for pelvic for all variation in different directions from isocenter position. The discoveries of our examination were in accordance with Engelsman $M$, et al., study. ${ }^{{ }^{11}}$ The greatness of precise and irregular mistakes for pelvic cancer cases in our investigation are comparable or not exactly different examinations, while we didn't utilize knee backing and footstool for pelvic and prostate disease patients. ${ }^{12-14}$ To contrast the outcomes and other distributed investigation, we have considered PTV (90, 95,) margin. In our investigation, the determined CTV-PTV edge for pelvis cancer patients in the vertical, longitudinal and lateral directions were $4.12 \mathrm{~mm}, 4.62 \mathrm{~mm}$ and 3.23 $\mathrm{mm}$, individually.

\section{CONCLUSION}

Translational variations measured in this study are in general agreement with previous studies. The use of the EPID in this study was less intrusive and may have resulted in less additional attention being given each imaging setup where the consequences of this examination are distinctive to the discoveries of current work. In the present work, we have changed the iso-center in the fourth portion, which brought about decrease of the efficient mistakes. Another distinction can be ascribed to the recurrence of online confirmation.

The margin to be added to the clinical target volume (CTV) to account for setup uncertainties will depend on whether it is possible to identify patients with significant translational variations, and to eliminate these displacements from routine treatments. The choice to eliminate these variations and to use a smaller CTV margin will have to be accompanied by stringent frequent position verification methods and repositioning.

\section{REFERENCES}

1. Dickerson RE, Haus AG, Huff KE . Development of a novel high-contrast cassette/film/screen system for radiationtherapy portal localization imaging. Proc SPIE. 1997; 3032:520-529.

2. Graff P, Hu W, Yom S S, Pouliot J: Does IGRT ensure target dose coverage of head and neck IMRT patients? RadiotherOncol, 2012;104:83-90.

3. Haus, AG. Through the Portal. Advance for Administrators in Radiology \& Radiation Oncology. 2000; 10(10):63-67.

4. Haus, AG, Dickerson RE, Huff KE, et al. Evaluation of a cassette-screen film combination for radiation therapy portal localization imaging with improved contrast. Med Phys. 1997;24(10): 16051608.

5. Oh YK, Baek JG, Kim OB, Kim JH (2014) Assessment of setup uncertainties for various tumor sites when using daily CBCT for more than 2200 VMAT treatments. Journal of applied clinical medical physics. 15(2): 4418 .

6. Padhani AR, Khoo VS, Suckling J, Husband JE, Leach MO, et al. (1999) Evaluating the effect of rectal distension and rectal movement on prostate gland position using cine MRI. Int $\mathrm{J}$ RadiatOncolBiolPhys 44(3): 525-533.

7. Dawson LA, Litzenberg DW, Brock KK, Sanda M, Sullivan M, et al. (2000) A comparison of ventilatory prostate movement in four treatment positions. Int J RadiatOncolBiolPhys 48(2): 319323.

8. Van Kranen S, van Beek S, MencarelliA ,RaschC,vanHerk M, SonkeJJ :Correction strategies to managed formations in head-and-neck radiotherapy. Radiother. Oncol,2010; 94:199-205.

9. Zhang L, Garden A S ,Lo J, et al., Multiple regionsof-interest analysis of setup uncertainties for headand-neck cancer radiotherapy. Int J RadiatOncolBiol Phys. 2006; 64:1559-1569.

10. Hunt, M. A., Schultheiss, T. E., Desobry, G. E., Hakki, M., \& Hanks, G. E. An evaluation of setup uncertainties for patients treated to pelvic sites. International Journal of Radiation Oncology, Biology, Physics, 1995;32(1),227-233. https://doi.org/10.1016/0360-3016(94)00 E.

11. Engelsman M, Rosenthal SJ, Michaud SL, et al. Intra- and inter-fractional patient motion for a variety of immobilization devices. Med Phys. 2005;32(11):3468-74.

12. Salter BJ, Fuss M, Vollmer DG, et al. The TALON removable head frame system for stereotactic radiosurgery/radiotherapy: measurement of the repositioning accuracy. Int $\mathrm{J}$ RadiatOncolBiol Phys. 2001;51(2):555-62.

13. Kooy HM, Dunbar SF, Tarbell NJ, et al. Adaptation and veri $\square$ cation of the relocatable GillThomas-Cosman frame in stereotactic radiotherapy. Int $\mathrm{J}$ RadiatOncolBiol Phys. 1994;30(3):685-91.

14. Van Santvoort J, Wiggenraad R, Bos P. Positioning accuracy in stereotactic radiotherapy using a mask system with added vacuum mouth piece and stereoscopic $\mathrm{x}$-ray positioning. Int $\mathrm{J}$ RadiatOncolBiol Phys. 2008;72(1):26. 